OPEN ACCESS

Edited by:

Qixin Zhou,

University of Akron, United States

Reviewed by:

Yalong Wang,

Hainan University, China

Xiaohuan Sun,

Yangzhou University, China

${ }^{*}$ Correspondence:

Meng Zheng

zhengmeng.555@hotmail.com

tThese authors have contributed equally to this work

Specialty section:

This article was submitted to

Organic Chemistry,

a section of the journal

Frontiers in Chemistry

Received: 29 June 2021

Accepted: 11 August 2021

Published: 25 August 2021

Citation:

Deng Z, Wang C, Li J and Zheng M (2021) Efficient Colorimetric Fluoride Anion Sensor Based on $\pi$-Conjugated

Carbazole Small Molecule.

Front. Chem. 9:732935.

doi: 10.3389/fchem.2021.732935

\section{Efficient Colorimetric Fluoride Anion Sensor Based on $\pi$-Conjugated Carbazole Small Molecule}

\author{
Zhifeng Deng ${ }^{1 \dagger}$, Cheng Wang ${ }^{2 \dagger}$, Junqiang $L^{3}{ }^{3}$ and Meng Zheng ${ }^{2,3 *}$ \\ ${ }^{1}$ National and Local Joint Engineering Laboratory for Slag Comprehensive Utilization and Environmental Technology, School of \\ Materials Science and Engineering, Shaanxi University of Technology (SNUT), Hanzhong, China, ${ }^{2}$ Key Laboratory of \\ Rubber-Plastic of Ministry of Education (QUST), School of Polymer Science and Engineering, Qingdao University of Science and \\ Technology, Qingdao, China, ${ }^{3}$ Qingdao Haiwan Science and Technology Industry Research Institute Co. Ltd., Qingdao, China
}

The ability to detect fluoride anions with high selectivity and sensitivity by using the naked eye is crucial yet challenging. In this study, a novel, simple conjugated organic dye, $\mathrm{N}$-tertbutyldimethylsilyl-3,6-diiodocarbazole (CA-TBMDS) was developed and used for the first time as a colorimetric sensor for fluoride. CA-TBMDS was found to be a highly sensitive fluoride chemosensor, with a detection limit as low as $3 \times 10^{-5} \mathrm{M}$. The reaction of CATBMDS with fluoride anions in a tetrahydrofuran solution resulted in a color change from colorless to yellow under ambient light, which can be discriminated by the naked eye. The sensor operated via intermolecular proton transfer between the amide units and the fluoride anion, as confirmed by proton nuclear magnetic resonance titration. CA-TBMDS is not only highly sensitive to fluoride anions, but also exhibits high sensitivity in the presence of various ions. This work demonstrates that $\mathrm{N}$-butyldimethylchlorosilane-based organic dyes have prospective utility as a type of fluoride anion chemosensor.

Keywords: fluoride anion sensors, naked eye detection, carbazole, intermolecular proton transfer, color change

\section{INTRODUCTION}

Fluoride is among the most electronegative ions and is the smallest anion, with a high charge density. Fluoride plays a key role in human health and chemical engineering because: (i) the fluoride anion is easily absorbed by the animal or human body, but it is excreted slowly. As a result, people or animals develop bone and thyroid activity disorders if they are overexposed to fluoride (Wade et al., 2010). (ii) Fluoride anions play a crucial role in organic synthesis, the chemical industry, biological and medical processes, and the military fields (Kleerekoper, 1998; Cametti and Rissanen, 2009; Wade et al., 2010; Xuan et al., 2013; Zhou et al., 2014; Li et al., 2018). An appropriate amount of fluoride anions in the environment is healthy for humans. However, a large amount of fluoride in the environment is hazardous and even toxic (Kaur and Choi, 2015). With the rapid development of the chemical industry, fluoride anions are present not only in aqueous environments, but also in organic media, such as waste organic liquor (Clark, 1980). The development of highly sensitive and selective fluoride anion sensors capable of qualitative and quantitative detection is crucial and could provide a diversity of optical chemosensors for fluoride anions in organic solutions.

In the past few years, many scientific studies have focused on the development of novel fluoride anion sensors with high sensitivity and selectivity (Yang et al., 2013; Feng et al., 2018; Wang et al., 2018; Antonio et al., 2020). Very recently, aminobenzodifuranone dyes for $\mathrm{F}^{-}$chemosensors were developed by our group, which could not only detect $\mathrm{F}^{-}$, but could also distinguish it from $\mathrm{F}^{-}$ containing solvents (Deng et al., 2020). Yuan et al. developed a new (3Z, 3'Z)-3,3'-(4,4,9,9-tetrakis 

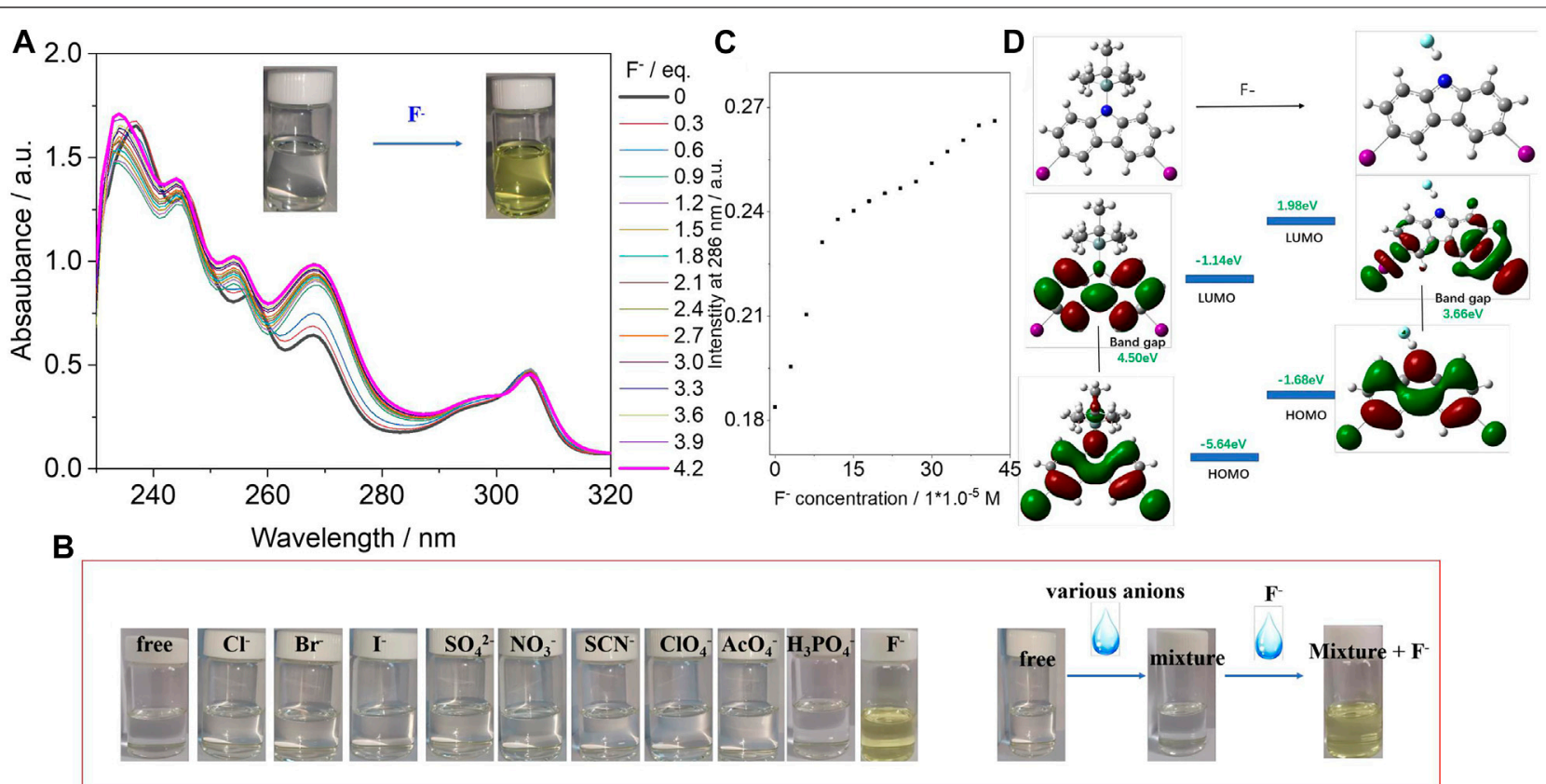

FIGURE 1 | (A) UV/vis absorption spectra of CA-TBMDS $\left(1.0 \times 10-{ }^{4} \mathrm{M}\right)$ in the presence of $\mathrm{F}^{-}(0-4.2$ eq. $)$ in THF. Inset shows photograph of CA-TBMDS solution with or without adding different amounts of fluoride anion under ambient light. (B) Photographs of CA-TBMDS $\left(5 \times 10^{-5} \mathrm{M}\right)$ in THF before and after adding different anions, as well the addition of other anions together with and without $\mathrm{F}^{-}$. (C) Optical absorption intensity at $286 \mathrm{~nm}$ against the concentration of fluoride anion. (D) Molecular orbital surfaces of the HOMO and LUMO energy levels of CA-TBMDS at the B3LYP/6-31G (d, p) level, before and after adding fluoride anion.

(4-hexylphenyl)-4,9-dihydro-s-indaceno [1,2-b:5,6-b']dithiophene)2,7-diylbis (methan-1-yl-1-ylidene))bis (6-bromo-indolin-2-one) (IDTI) dye with a detection limitation as low as $1 \times 10^{-7} \mathrm{M}$ for the fluoride anion (Yuan et al., 2020). Additionally, Zhang and coworkers developed a DPP-based polymer from $t$-butoxy carbonyl $(t$-Boc) units that detect fluoride anions, and also extract fluoride anions from organic solutions (Zhang et al., 2018).

The reported fluoride anion sensors generally react with fluoride anions, resulting in changes in the UV-vis absorption and/or fluorescence emission spectra of the sensors. Among these, the sensors that allow for the detection of color with the naked eye are more interesting and promising because fluoride anions can be detected easily and simply without the need for auxiliary equipment. Herein, a new organic conjugated carbazole small molecule, which can allow for fluoride detection with the naked eye, was developed for use as a fluoride anion chemosensor.

\section{MATERIALS AND METHODS}

\section{N-Tert-Butyldimethylsilyl- \\ 3,6-Diiodocarbazole (CA-TBMDS)}

In a dry flask under nitrogen protection, 3,6-diiodocarbazole $(2.00 \mathrm{~g}, 4.77 \mathrm{mmol})$ was dissolved in anhydrous tetrahydrofuran (THF; $30 \mathrm{ml}$ ) at room temperature. Sodium hydride $(172 \mathrm{mg}$, $7.2 \mathrm{mmol}$ ) was added to the stirred solution, which was further stirred at room temperature for another $30 \mathrm{~min}$ tertButyldimethylsilyl chloride $(0.79 \mathrm{~g}, 5.3 \mathrm{mmol})$ was then added and the reaction was stirred at room temperature for a further $17 \mathrm{~h}$. The reaction mixture was then poured into ice water $(50 \mathrm{ml})$ and extracted three times with dichloromethane. The combined organic layers were dried over $\mathrm{MgSO}_{4}$ before the solvent was removed in vacuo, which afforded an off-white solid. The crude product was purified through a plug of silica in a fitted funnel with hexane/dichloromethane (9:1) as the eluent. After removing the solvent in vacuo, the product was obtained (2.06 g, yield: $81 \%) .{ }^{1} \mathrm{H}$ NMR $\left(500 \mathrm{MHz}, d_{6}\right.$-DMSO) $\delta \mathrm{ppm}: 8.61(\mathrm{~s}, 2 \mathrm{H})$, $7.65-7.67(\mathrm{~d}, \mathrm{~J}=10 \mathrm{~Hz}, 2 \mathrm{H}), 7.51-7.53(\mathrm{~d}, \mathrm{~J}=10 \mathrm{~Hz}, 2 \mathrm{H})$, $0.96(\mathrm{~s}, 9 \mathrm{H}), 0.75(\mathrm{~s}, 3 \mathrm{H})$.

\section{RESULTS AND DISCUSSION}

The synthesis of CA-TBMDS is described in the Materials and Methods section. CA-TBMDS showed good solubility in most common organic solvents. The interaction between the CATBMDS chromophore and fluoride anions was first investigated by using the naked eye to determine the color change. As shown in the inset of Figure $\mathbf{1 A}$, the pure CATBMDS solution was colorless. Once the fluoride anion was introduced, the color of the solution immediately changed to yellow. This indicated that in the presence of CA-TBMDS, fluoride anions could be detected by the naked eye without additional equipment. Additionally, CA-TBMDS presented blue emission, which is sensitive to the naked eyes. A spectrophotometric titration was used to investigate the interaction between the CA-TBMDS chromophore and 


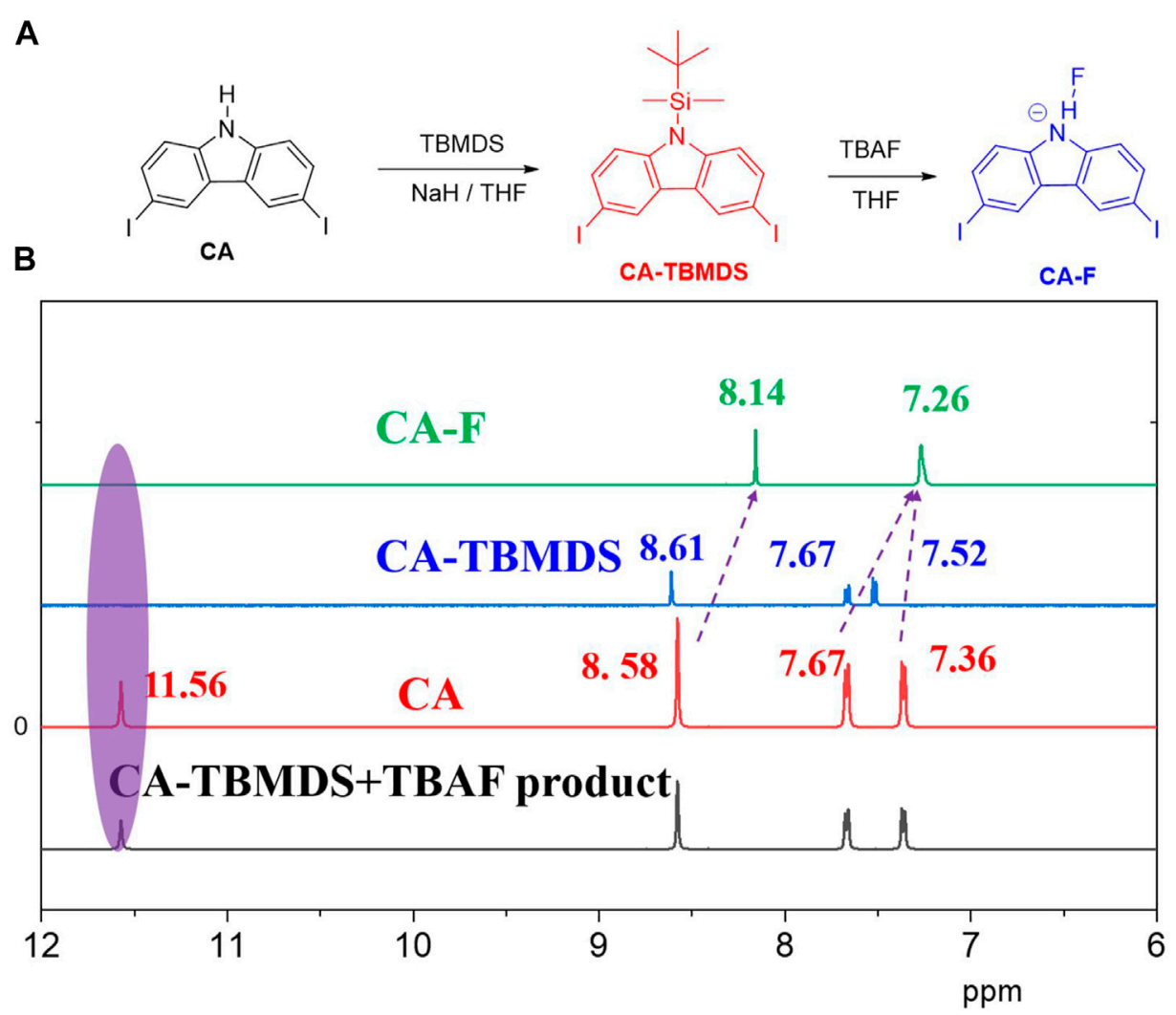

FIGURE 2 | (A) Synthesis of CA-TBMDS, and reaction between CA-TBMDS and fluoride anion. (B) Partial ${ }^{1} \mathrm{H}$ NMR titration spectra of CA and CA-TBMDS before and after adding fluoride anion (4 eq.) in DMSO- $d_{6}$.

fluoride anion in THF solution. A standard solution of tetrabutylammonium fluoride (TBAF, $\left.1.0 \times 10^{-2} \mathrm{M}\right)$ was gradually added to a $1.0 \times 10^{-4} \mathrm{M}$ solution of CA-TBMDS in THF. As shown in Figure 1A, with the progressive addition of fluoride anions, the absorption intensity at $306 \mathrm{~nm}$ showed almost no change, but other absorption peaks were slightly blue-shifted, which enhanced the absorption intensity. For example: (i) the absorption peak at $268 \mathrm{~nm}$ with an intensity of 0.61 shifted to $267 \mathrm{~nm}$ with an intensity of 0.99 ; (ii) the absorption peak at $256 \mathrm{~nm}$ with an intensity of 0.80 shifted to $254 \mathrm{~nm}$ with an intensity of 1.01; (iii) the absorption peak at $245 \mathrm{~nm}$ with an intensity of 1.27 shifted to $244 \mathrm{~nm}$ with an intensity of 1.38; (iv) the absorption peak at $236 \mathrm{~nm}$ with an intensity of 1.31 shifted to $234 \mathrm{~nm}$ with an intensity of 1.70 . In addition, the optical absorption intensity of the spectra vs the concentration of fluoride anion was calculated which was described in Figure 1C. Figure 1C showed that the detection limit of CA-TBMDS for the fluoride anions was at least $3 \times 10^{-5} \mathrm{M}$. As shown in Figure $\mathbf{1 B}$, apart from $\mathrm{F}^{-}$(as tetrabutylammonium salts), anions (4 equivalents) such as $\mathrm{Cl}^{-}$, $\mathrm{Br}^{-}, \mathrm{I}^{-}, \mathrm{SO}_{4}{ }^{2-}, \mathrm{NO}_{3}{ }^{-}, \mathrm{SCN}^{-}, \mathrm{ClO}_{4}^{-}, \mathrm{AcO}_{4}^{-}$, and $\mathrm{H}_{2} \mathrm{PO}_{4}{ }^{-}$caused almost no change in the color of the CA-TBMDS solution. Interestingly, there was no noticeable color change upon the addition of other anions together with $\mathrm{F}^{-}$, although $\mathrm{F}^{-}$by itself led to an immediate change from colorless to yellow (Figure 2B).
This observation indicates that fluoride anions can be detected without interference from other anions. Thus, CA-TBMDS seems to be a highly sensitive and selective sensor for fluoride anions.

The changes in the color and optical absorption spectrum are plausibly associated with the decomposition of $t$-butyldimethylchlorosilane (TBMDS) from CA-TBMDS. Once CA-TBMDS interacted with the fluoride anion, the CA-TBMDS molecules were transformed to CA, which generated N-H units. This was confirmed by the NMR test. The CA-TBMDS solution was added by TABF solution, subsequently the mixture was stirred for $15 \mathrm{~min}$ under room temperature. The mixture was purified by the saturated $\mathrm{NH}_{4} \mathrm{Cl}(\mathrm{aq})$ and extracted with toluene. The combined organic layers were washed with deionized water then dried by $\mathrm{MgSO}_{4}$ before the solvent was removed in vacuo to afford the white product. The NMR of the product was measured, which showed the same peaks compared to the CA. Compare to the CA-TBMDS, the peaks of the product were shifted into slightly lower ppm, for instance the proton of the carbazone core of $8.61 \mathrm{ppm}$ shifted into $8.58 \mathrm{ppm}$, while $7.72 \mathrm{ppm}$ was moved to $7.36 \mathrm{ppm}$. The lactam $\mathrm{NH}$ moiety from the CA core was able to interact with the fluoride anions, which easily deprotonated the $-\mathrm{NH}$ protons (inter-molecular proton transfer, IPT, Figure 2A (Zhang et al., 2018)). ${ }^{1} \mathrm{H}$ NMR experiments were carried out in DMSO- $d_{6}$ to confirm our assumption and further understand the interaction between 
the fluoride anion and the CA-TBMDS acceptor. As shown in Figure 2B, except for the signal of the amino proton at $11.56 \mathrm{ppm}$, the specific signals from the carbazole core of CA and CA-TBMDS were similar. Once the fluoride anions were added, the proton signal of carbazole at $8.58 \mathrm{ppm}$ shifted to 8.14 ppm, while the respective proton signals at 7.67 and $7.36 \mathrm{ppm}$ shifted to $7.26 \mathrm{ppm}$. This may be due to the IPT process, which severed the TBMDS units from the CA core, and resulted in hydrogen bonding between the fluoride anion and the proton on the amino $\mathrm{N}-\mathrm{H}$. In addition, the signals of the amino protons at $11.56 \mathrm{ppm}$ for CA did not appear, which further confirmed this assumption.

To further understand the electron distributions before and after fluoride anion binding, the Frontier molecular orbital (FMO) energy was calculated at the B3LYP/6-31 (d, p) level using CA-TBMDS. As shown in Figure 1D, the distribution of the highest occupied molecular orbital (HOMO) and lowest unoccupied molecular orbital (LUMO) orbitals of CA-TBMDS was similar because these orbitals were mainly localized on the core of CA. After binding with the fluoride anions, the HOMO orbital distributions showed almost no change, but the electron distribution of the LUMO, which was associated with the binding of CA to the fluoride anions, was mainly located at both ends of the CA core. This indicates that when the CA became excited, electron transfer from the core of CA to both ends took place at the fluoride-bonded CA. In addition, after binding with the fluoride anions, the bandgap of the molecules decreased.

\section{CONCLUSION}

A novel colorimetric chemosensor for detecting fluoride anions was designed and studied. This novel sensor, CA-TBMDS, based on carbazole, exhibits high sensitivity and selectivity for fluoride. CA-TBMDS reacts with fluoride anions in organic solvents, resulting in a visible color change from colorless to yellow, which can be detected with the naked eye. The color change is associated with severance of the TBMDS units from CA-TBMDS by the fluoride anion and simultaneous formation of $\mathrm{NH}$ units,

\section{REFERENCES}

Cametti, M., and Rissanen, K. (2009). Recognition and Sensing of Fluoride Anion. Chem. Commun. 28, 2809-2829. doi:10.1039/ b902069a

Clark, J. H. (1981). ChemInform Abstract: FLUORIDE ION AS A BASE IN ORGANIC SYNTHESIS. Chemischer Informationsdienst. 12, 80429-80452. doi:10.1002/chin.198121368

Deng, Z. F., Li, R., Geng, J. T., Zheng, M., Li, L. Q., Shi, X., et al. (2020). Efficient Colorimetric Fluoride Anion Chemosensors with Varied Colors Based on Simple Aminobenzodifuranone Organic П-Conjugated Dyes. Front. Chem. 8, 231. doi:10.3389/ fchem.2020.00231

Feng, Y., Li, X., Ma, H., Zhang, Z., Zhang, M., and Hao, S. (2018). A Simple Fluorescent Film Probe for the Detection of Fluoride Anion in Organic Solution. Dyes Pigm. 153, 200-205. doi:10.1016/ j.dyepig.2018.02.004 leading to intermolecular proton transfer between CATBMDS and the fluoride anions. Spectroscopic studies show that for CA-TBMDS, the detection limit for the fluoride anion was as low as $3 \times 10^{-5} \mathrm{M}$. This work demonstrates that CA-TBMDS, with its high sensitivity and selectivity, is a promising dye for fluoride chemosensors, enabling naked eye detection of target analytes. In addition, N-TBMDS units containing organic dyes can be used to produce fluoride anion sensors.

\section{DATA AVAILABILITY STATEMENT}

The original contributions presented in the study are included in the article/Supplementary Material, further inquiries can be directed to the corresponding author.

\section{AUTHOR CONTRIBUTIONS}

$\mathrm{ZD}$ and $\mathrm{CW}$ carried out in experiment and prepared the article. $\mathrm{MZ}$ and JL supervised the whole work. All authors discussed and commented on the paper.

\section{FUNDING}

This study was financially supported by the National \& Local Joint Engineering Laboratory for Slag Comprehensive Utilization and Environmental Technology Open Fund (SLGPT2019KF0101), Research Projects of Shaanxi University of Technology (SLG 1901) and Qingdao Postdoctoral Applied Research Project of Shandong Province (No. QDBSHYYYJXM202001-28).

\section{SUPPLEMENTARY MATERIAL}

The Supplementary Material for this article can be found online at: https:/www.frontiersin.org/articles/10.3389/fchem.2021.732935/ full\#supplementary-material

Kaur, M., and Choi, D. H. (2015). Diketopyrrolopyrrole: Brilliant Red Pigment Dye-Based Fluorescent Probes and Their Applications. Chem. Soc. Rev. 44, 58-77. doi:10.1039/c4cs00248b

Kleerekoper, M. (1998). The Role of Fluoride in the Prevention of Osteoporosis. Endocrinol. Metab. Clin. North America. 27, 441-452. doi:10.1016/S08898529(05)70015-3

Li, M., Liu, Z., Wang, H.-C., Sedgwick, A. C., Gardiner, J. E., Bull, S. D., et al. (2018) Dual-function Cellulose Composites for Fluorescence Detection and Removal of Fluoride. Dyes Pigm. 149, 669-675. doi:10.1016/j.dyepig.2017.11.033

Sánchez-Ruiz, A., González-Alfaro, S., García-Martínez, J. C., and Rodríguez-López, J. (2020). A Study of Silylated Tris(styryl)benzenes as Potential Fluorescent Sensors for Aqueous Fluoride. Dyes Pigm. 182, 108610. doi:10.1016/j.dyepig.2020.108610

Wade, C. R., Broomsgrove, A. E. J., Aldridge, S., and Gabbaï, F. P. (2010). Fluoride Ion Complexation and Sensing Using Organoboron Compounds. Chem. Rev. 110, 3958-3984. doi:10.1002/chin.20104125010.1021/cr900401a

Wang, R., Li, J., Li, G., Hao, C., Zhang, Y., Wang, S., et al. (2018). Synthesis of 1-Amino12-Hydroxyl-Perylene Tetra-(alkoxycarbonyl) for Selective Sensing of Fluoride. Dyes Pigm. 156, 225-232. doi:10.1016/j.dyepig.2018.04.012 
Xuan, W., Cao, Y., Zhou, J., and Wang, W. (2013). A FRET-Based Ratiometric Fluorescent and Colorimetric Probe for the Facile Detection of Organophosphonate Nerve Agent Mimic DCP. Chem. Commun. 49, 10474-10476. doi:10.1039/c3cc46095a

Yang, C., Zheng, M., Li, Y., Zhang, B., Li, J., Bu, L., et al. (2013). N-monoalkylated 1,4Diketo-3,6-Diphenylpyrrolo[3,4-C]pyrroles as Effective One- and Two-Photon Fluorescence Chemosensors for Fluoride Anions. J. Mater. Chem. A. 1, 5172-5178. doi:10.1039/C3TA00160A

Yuan, X., Shi, X., Wang, C., Du, Y., Jiang, P., Jiang, X., et al. (2020). IDTI Dyes for Fluoride Anion Chemosensors. Front. Chem. 8, 591860. doi:10.3389/ fchem.2020.591860

Zhang, H., Yang, K., Chen, C., Wang, Y., Zhang, Z., Tang, L., et al. (2018). 1,4Diketo-pyrrolo[3,4-c]pyrroles (DPPs) Based Insoluble Polymer Films with Lactam Hydrogens as Renewable Fluoride Anion Chemosensor. Polymer 149, 266-272. doi:10.1016/j.polymer.2018.07.011

Zhou, Y., Zhang, J. F., and Yoon, J. (2014). Fluorescence and Colorimetric Chemosensors for Fluoride-Ion Detection. Chem. Rev. 114, 5511-5571. doi: $10.1021 / \mathrm{cr} 400352 \mathrm{~m}$
Conflict of Interest: MZ and JL belong to the commercial affiliation of Qingdao Haiwan Science and Technology Industry Research Institute Co., Ltd. declare that the research was conducted in the absence of any commercial or financial relationships that could be construed as a potential conflict of interest.

Publisher's Note: All claims expressed in this article are solely those of the authors and do not necessarily represent those of their affiliated organizations, or those of the publisher, the editors and the reviewers. Any product that may be evaluated in this article, or claim that may be made by its manufacturer, is not guaranteed or endorsed by the publisher.

Copyright (c) 2021 Deng, Wang, Li and Zheng. This is an open-access article distributed under the terms of the Creative Commons Attribution License (CC $B Y)$. The use, distribution or reproduction in other forums is permitted, provided the original author(s) and the copyright owner(s) are credited and that the original publication in this journal is cited, in accordance with accepted academic practice. No use, distribution or reproduction is permitted which does not comply with these terms. 